\title{
Evaluation of Pre-Operative Risk Factors for Post-Surgical Wound Infection in Elective Operation
}

\author{
Md. Shahidul Islam, ${ }^{7}$ Mitheel-Ibna Islam, ${ }^{2}$ Md. Jamal Saleh Uddin, ${ }^{3}$ Abul Kalam Md. Faruq, ${ }^{4}$ \\ Samar Chandra Saha, Shitil Ibna Islam ${ }^{6}$
}

\begin{abstract}
Background \& Objective: Postoperative surgical site infections (SSIs) are still among the most common serious complications of surgery in terms of cost and consequences. Different risk factors like age, sex, nutrition, immunity, operation type, duration of stay in hospital and presence of hair on the skin may involve with SSIs. This study was intended to determine the risk factors for surgical site infections in a tertiary level hospital.

Methods: This Cross Sectional observational study was carried out in the Department of Surgery, Rajshahi Medical College Hospital, Rajshahi, over a period of 12 months from May 2009 to April 2010. A total of 232 patients (aged 18 years onwards) of either sex scheduled for elective operation were included in the study. Common indications for operation were chronic calculus cholecystitis, stoma in situ, inguinal hernia, renal/urinary stones and benign prostatic hyperplasia and so on. As two patients were discharged on request on $2^{\text {nd }}$ postoperative day, 230 patients were left for final analysis. The patients were followed till discharge.

Results: Of the total 230 patients, $21(9.1 \%)$ developed post-operative wound infection. The incidence of wound infection was significantly higher in older patients $(p=0.014)$. However sex did not have any influence on wound infection. The malnourished patients (underweight in terms of BMI) developed SSIs more frequently than their normal or overweight counterparts $(p<0.001)$. Diabetic patients developed post-operative wound infection more often than the non-diabetics $(p=0.072)$. The incidence of post-operative wound infection was not found to be associated with shaving of hair on the skin over the operative site $(p=0.378)$. Contaminated wounds encountered SSI significantly more than the clean wounds did ( $p=0.001)$.

Conclusion: The study concluded that one in every ten patients undergoing abdominal surgery may develop postoperative wound infection and factors commonly associated with wound infection are older age, underweight, diabetes, contaminated wound, longer preoperative and postoperative hospital stay. The study suggests that reducing the factors of SSI (as far as practicable) will reduce the incidence of SSI.

Key words: Surgical site infections, elective intra-abdominal operation, risk factors etc.
\end{abstract}

\section{INTRODUCTION}

Before the mid-19th century, surgical patients commonly developed postoperative wound infection followed by purulent discharge from their incisions and overwhelming sepsis often led to death. With the introduction of principles of asepsis in the late 1860 s by Joseph Lister, postoperative infectious morbidity and mortality decreased substantially. Further advancement in infection control was facilitated by improved

\footnotetext{
Authors' information:

${ }^{1}$ Dr. MD. Shahidul Islam, FCPS (Surgery), Junior Consultant, 250 Bed Shaheed Sheikh Abu Naser Specialized Hospital, Khulna.

${ }^{2}$ Dr. Mitheel-Ibna Islam, FCPS (Obstetrics \& Gynaecology), DGO, Medical Officer, 250 Bed Shaheed Sheikh Abu Naser Specialized Hospital, Khulna.

${ }^{3}$ Dr. Md. Jamal Saleh Uddin, MS (Paediatric surgery), Associate Professor, Paediatric Surgery, Comilla Medical College, Comilla.

${ }^{4}$ Dr. Abul Kalam Md. Faruq, BDS, MS (OMS), Assistant Professor, Oral \& Maxillofacial Surgery, Dhaka Dental College, Dhaka.

${ }^{5}$ Dr. Samar Chandra Saha, Registrar, Department of Anesthesiology \& ICU, Holy Family Red Crescent Medical College Hospital, Dhaka

${ }^{6}$ Shitil Ibna Islam, Research Officer, Ibrahim Cardiac Hospital \& Research Institute, Shahbag, Dhaka.
}

Correspondence: Dr. MD. Shahidul Islam, Cell phone:+8801711-787624 E-mail:mukul.mi86@gmail.com 
operating room ventilation, sterilization methods, surgical technique and availability of antimicrobial prophylaxis. Despite these activities, surgical site infections (SSIs) still remain the third most frequently reported nosocomial infection in terms of morbidity, mortality, ${ }^{1}$ postoperative hospital stay and hospital $\operatorname{cost}^{2,3}$ This persistently significant rate of SSI may be partially explained by the emergence of antimicrobial-resistant pathogens and the increased numbers of surgical patients who are elderly and/or have a wide variety of chronic, debilitating or immunocompromising underlying diseases. Cruse and Foord, ${ }^{4}$ in a large prospective study of surgical site infections, identified diabetes mellitus, obesity, malnutrition, chronic renal failure, advanced age and use of steroids as risk factors for increased wound infection rates. All of these risk factors may delay wound healing by influencing inflammation and synthesis of new wound matrix and collagen. ${ }^{5}$ Therefore the identification of host and environmental factors that increase the likelihood of a poor outcome, as well as appropriate patient preparation, timely and meticulous closure of wounds are of utmost importance in the prevention of surgical site infection.

The Department of Surgery in Rajshahi Medical College Hospital (RMCH) is overburdened with a huge number of elective and emergency operations being done in the department each year. The department is managed by only fewer postgraduate surgeons, supported by about twenty postgraduate trainees and forty intern doctors. As a consequence, a significant proportion of operations are done by trainee doctors who are not fully adherent to principles of asepsis and sound surgical techniques. Due to extreme workload, nurses also fail to maintain principles of asepsis. In $\mathrm{RMCH}$ all patients undergoing surgery receive prophylactic antibiotics. But there is no protocol regarding choice of antimicrobial agents. The operating room is frequently entered by doctors and students of various levels. Third and subsequent operations of a day are often done by inadequately sterilized instruments. Most of the patients seldom maintains personal hygiene. Wards are always overcrowded by patients' attendants. All these limitations might have led to a higher rate of post-operative wound infection in the hospital which results in extended and unexpected hospital stay, adds burden of pain and suffering to the patients and their relatives, increases cost for the individuals and hospitals, and deprives of other patients deserving treatment most. Healing of an infected wound produces an ugly scar. Some patients subsequently develop incisional hernia. Thus, to reduce incidence of SSI, it is important to identify the risk factors that increase the incidence. This issue has been addressed in many centers in the world with differing results and recommendation. It has been found that SSI surveillance and institutional implementation of protocol targeting known risk factors reduce wound infection significantly. ${ }^{6-8}$ In Bangladesh very few studies regarding the risk factors for SSIs have yet been conducted. The present study, intended to identify individuals with particular characteristics or underlying medical conditions could predispose the development of SSIs, is therefore of utmost significance and will help deciding antibiotic therapy and additional care to be needed to expedite the process of recovery.

\section{PATIENTS \& METHODS}

This Cross Sectional observational study was carried out in the Department of Surgery, Rajshahi Medical College Hospital, Rajshahi, over a period of 12 months from May 2009 to April 2010. A total of 232 patients (aged 18 years onwards) of either sex scheduled for elective operation were included in the study. Common indications for operation were chronic calculus cholecystitis, stoma in situ, inguinal hernia, renal/urinary stones, benign prostatic hyperplasia and so on (Table I). As two patients were discharged on request on $2^{\text {nd }}$ postoperative, 230 patients were left for final analysis. The outcome variable was SSI or wound infection, which was defined as pain, swelling, redness with or without discharge of pus at the surgical site or dehiscence of wound. The patients were followed till discharge. The statistical 
analyses were performed using computer software SPSS (Statistical Package for Social Sciences), version 17 and the test statistics used to analyze the data were Student's t-Test and Chi-square Test. The level of significance was set at $5 \%$ and $\mathrm{p}<0.05$ was considered that the factors concerned were significantly associated with surgical site infection.

\section{RESULT}

Over $70 \%$ of the patients were $>50$ years old with mean age of the patients being $44.5 \pm 16.5$ years (range: 14-90 years). Sixty percent of the patients were male. Of the total patients, $21(9.1 \%)$ developed post-operative wound infection. The incidence of wound infection was significantly higher in older age group ( $p=0.014)$, but there was no significant association between sex and postoperative wound infection $(p=0.513)$. The malnourished patients (underweight in terms of BMI) developed SSIs more frequently than their normal or overweight counterparts ( $p<0.001$ ). Diabetic patients developed post-operative wound infection more often than the non-diabetics $(p=0.072)$. The incidence of post-operative wound infection was not found to be associated with shaving of hair on the skin over the operative site $(p=0.378)$.

\section{TABLE I. Common indications for operation $(n=230)$.}

\begin{tabular}{|c|c|c|}
\hline Clinical diagnosis & List of operation & patients \\
\hline Ch. Cal.Cholecystitis & Open Cholecystectomy & $34(14.8)$ \\
\hline Stoma in situ & Closure of the Stoma & $32(13.9)$ \\
\hline Inguinal Hernia & Herniorrhaphy/Hernioplasty & $31(13.5)$ \\
\hline Renal/Ureteric Stone & Pyelolithotomy/Ureterolithotomy etc. & $22(9.6)$ \\
\hline $\mathrm{BPH}$ & Open Prostatectomy & $20(8.7)$ \\
\hline Colorectal Cancer & Hemicolectomy/APRIAnt. Resection & $10(4.3)$ \\
\hline Incisional Hernia & Hernioplasty & $9(3.9)$ \\
\hline Gastric Carcinoma & Radical Gastrectomy & $7(3.0)$ \\
\hline Pancreatic Disease & PPPD/Drainage procedures & $7(3.0)$ \\
\hline Pyloric Stenosis & B.T. Vagotomy \& Gastrojejunostomy & $6(2.6)$ \\
\hline Vesical Stone & Suprapubic Cystolithotomy & $5(2.2)$ \\
\hline CBD Stone & Cholidocholithotomy & $5(2.2)$ \\
\hline Hydronephrosis & A-H Pyeloplasty/Nephrectomy & $4(1.7)$ \\
\hline Buerger's Disease & B.L. Symphathetectomy & $4(1.7)$ \\
\hline Renal Cancer & Nephrectomy & $4(1.7)$ \\
\hline Others & & $30(13.0)$ \\
\hline
\end{tabular}

As wound type was concerned, contaminated wounds encountered SSI significantly more often than the clean wounds did ( $p=0.001)$ (Table II).

\begin{tabular}{|c|c|c|c|}
\hline \multicolumn{4}{|c|}{$\begin{array}{l}\text { TABLE II. Association between patient characteristics and } \\
\text { surgical site of infections }\end{array}$} \\
\hline \multirow[b]{2}{*}{ Baseline characteristics } & \multicolumn{2}{|c|}{ Wound infection } & \multirow[b]{2}{*}{ p-value } \\
\hline & $\begin{array}{c}\text { Yes } \\
(n=21)\end{array}$ & $\begin{array}{c}\text { No } \\
(n=209)\end{array}$ & \\
\hline \multicolumn{4}{|l|}{ Age (years)* } \\
\hline$\leq 50$ & $10(47.6)$ & 153(73.2) & 0.014 \\
\hline$>50$ & $11(52.4)$ & $56(26.8)$ & \\
\hline \multicolumn{4}{|l|}{ Sex } \\
\hline Male & $14(66.7)$ & $124(59.3)$ & 0.513 \\
\hline Female & $7(33.3)$ & $85(40.7)$ & \\
\hline \multicolumn{4}{|l|}{ BMI } \\
\hline Under weight (up to 18.5 ) & $7(33.3)$ & $20(9.6)$ & \\
\hline Obese $(>30)$ & $2(9.5)$ & $10(4.8)$ & $<0.001$ \\
\hline Normal ( $18.5-30)$ & $12(57.2)$ & 179(85.6) & \\
\hline DM & $3(14.3)$ & $10(4.8)$ & 0.072 \\
\hline \multicolumn{4}{|l|}{ Hair removal pattern } \\
\hline None & $10(47.6)$ & $91(43.5)$ & \\
\hline Prior to surgery & $3(14.3)$ & $58(27.8)$ & 0.378 \\
\hline Day before surgery & $8(38.1)$ & $60(28.7)$ & \\
\hline \multicolumn{4}{|l|}{ Wound class } \\
\hline Clean & $3(14.3)$ & $60(28.7)$ & 0001 \\
\hline Contaminated & $18(85.7)$ & 137(71.3) & 0.001 \\
\hline
\end{tabular}

Figures in the parentheses indicate corresponding percentage; ${ }^{*}$ Chi-squared Test $\left(\chi^{2}\right)$ was done to analyze the data.

The mean duration of preoperative hospital stay was longer in patients who developed postoperative wound infection ( $18 \pm 10.3$ days) than those who did not develop ( $12.7 \pm 8.3$ days) ( $p=0.023$ ). Postoperative hospital stay was also much longer in patients who developed wound infection than those who did not develop the condition $(21.9 \pm 7.6$ vs. $8.5 \pm 4.1$ days, $p<$ 0.001 ) (Table III).

$\begin{aligned} & \text { TABLE II. Association between hospitalsstays and wound } \\ & \text { infection }\end{aligned}$
$\begin{array}{lccc}\text { Hospitals stays (days) } & \begin{array}{c}\text { Wound infection } \\ \text { Yes } \\ (n=21)\end{array} & \begin{array}{c}\text { No } \\ (n=209)\end{array} & \text { p-value } \\ & 18 \pm 10.3 & 12.7 \pm 8.3 & 0.023 \\ \text { Preoperative } & 21.9 \pm 7.6 .7 & 8.5 \pm 4.1 & <0.001 \\ \text { Postoperative } & \end{array}$

Data were analysed using Unpaired t-Test and were presented as mean $\pm S D$. 
Type of operations, commonly associated with SSIs, were gastric surgery, hernia repair, intestinal surgery, urological surgery etc. (Table IV). Postoperative wound infection was managed by regular dressing, change of antibiotic with or without culture and sensitivity test, fresh blood transfusion, secondary closure and reoperation as appropriate (Table V).

\begin{tabular}{|lcr|}
\hline \multicolumn{3}{|l}{ TABLE IV. Lists of operations with frequency and SSIsrates } \\
\hline Interventions & No of patients & No(\%) \\
\hline Gastric Surgery & 13 & $2(15.4)$ \\
Hernia Repair & 43 & $5(11.6)$ \\
Urological surgeries & 55 & $5(9.0)$ \\
Intestinal Surgery & 51 & $5(9.8)$ \\
Biliary Surgery & 44 & $4(9.0)$
\end{tabular}

TABLE IV. Management of postoperative wound infections $(n=21)$

$\begin{array}{lcc}\text { Treatment of SSI } & \text { No of patients } & \text { Percentage } \\ \text { Change of antibiotic } & 21 & 100 \\ \text { Culture and sensitivity test } & 8 & 38.0 \\ \text { Fresh blood transfusion } & 7 & 33.3 \\ \text { Secondary closure/mass closure } & 15 & 71.4 \\ \text { Reoperation } & 1 & 4.7\end{array}$

* Total will not corresponded to $100 \%$ for multiple response

\section{DISCUSSION}

The successful outcome after surgery is highly dependent on the incidence and severity of postoperative complications. Surgical site infection is the most common complications of intra-abdominal surgery. Post-operative wound infection not only increases morbidity, mortality, hospital stay and costs, but also compromises cosmetic outcome. The overall incidence of SSI in elective intra-abdominal surgery was $9.1 \%$. The apparently low incidence of SSI is due to selection of elective cases and exclusion of emergency procedures. The infection rate was significantly low for clean and uncontaminated wound than that for contaminated operation. Similar observation was reported in some other literatures, but Culver et al described much lower infection rate in all the three wound classes. ${ }^{6}$

In this series, common indications for operation in descending order include chronic calculus cholecystitis, stoma in situ, inguinal hernia, renal/urinary stones and benign prostatic hyperplasia. SSI was relatively higher following gastric surgery $(15.4 \%)$, hernia repair $(11.6 \%)$, and intestinal surgery $(9.8 \%)$. Of the 21 cases of SSI, superficial infection was found in $13(61.9 \%)$ cases, mere incisional tissue infection in $6(28.6 \%)$ cases and organ/space infection in $2(9.5 \%)$ cases.

Among the eight preoperative risk factors analysed, five identified for wound infection were older age ( $>50$ years), underweight, diabetes mellitus, longer preoperative hospital stay and contaminated surgical site. Age is a risk factor for postoperative infection complication, commonly reported in the medical literature. $7,9,10$ Increasing age is correlated with greater likelihood of certain chronic conditions, malnutrition and a fall in the body immunological efficiency, causing more extensive SSI. However, SSI was not found to be associated with sex, which is in agreement with previous findings. ${ }^{7-9,11-13}$ Nutritional state is regarded as an important factor in wound healing. ${ }^{14}$ Being underweight indicates poor nutritional status of patients. Body hair is conventionally removed from a proposed operative field the day before surgery. This is done for aesthetic reason and also to allow painless removal of dressings. It has been shown in various studies, however, that the time of shaving is crucial. When performed over 12 hours before surgery wound sepsis rates increase from $1-2 \%$ up to more than $5 \%$. Unsupervised shaving by patients worsens these rates.

The previously reported association between SSI and pre-operative bed stay-9, $62,15,16$ was marginally supported by this study ( $p<0.05)$. Longer pre-operative hospital stay increases the likelihood of colonization in patients with nosocomial strains of bacteria which are more resistant to antibiotic. Cruse and Foord $^{10}$ reported that longer stay in hospital indirectly increases infection rate by lowering patients' resistance. This is one of the factors to be taken into account to reduce the incidence of SSI. Longer postoperative stay also increases the likelihood of infection as was evidenced by the findings of the present study.

Sound surgical technique is widely believed to reduce 
the risk of SSI. Such techniques include maintaining effective homeostasis while preserving adequate blood supply, preventing hypothermia, gently handling tissue, avoiding inadvertent entries into a hollow viscous, removing devitalized (e.g. necrotic or charred) tissues, using drain and suture material appropriately, obliterating dead space, and appropriately managing the postoperative incision. Thus individual surgeon performing the procedure can have an impact on outcome of the patient. There is evidence to suggest that grade of surgeon can have an impact on SSI rate. ${ }^{15}$ Guidance from the Specialist Advisory Committee of the Joint Committee on Higher Surgical Training of Scotland suggests that where a consultant does not perform the procedure, one should be present in the operating theatre in order to minimize this risk. ${ }^{16}$

In our study, drain usage or type of drain have shown no impact on SSI rate, but presence of drain for longer than 3 days increased wound infection rate significantly. Similar observation was reported in medical literatures. ${ }^{8,14,17}$ In some studies, use of drain was found to be an independent risk factor for SSI. ${ }^{7,9,12,13}$ The main aim of drainage includes early detection of an unwanted event such as biliary leakage and to evacuate residual effusions to avoid infection. Thus, every drain should have its own limits because of its possibility of obstruction leading to retrograde bacterial colonization, especially when the drainage is no longer an aspirate.

In our study, contaminated wound was found to be significantly associated with post-operative wound infection. When the intrinsic contamination could not be expected to be minimized by modification in technique, emphasis should be placed on other factors strongly associated with the development of SSI, nutritional status, length of preoperative stay etc.

Despite utmost caution to avoid bias, several limitations were encountered. First, some variables found in other literatures as infectious risk factors such as immunodeficiency, operation theatre environment, skin infection, antibiotic prophylaxis, suture material and hypothermia, have not been studied. Second, the study did not have the scope of post-discharge SSI where post-operative hospital stay was too short ( $<5$ days).

Based on the findings of the study, it can be concluded that one every ten patients undergoing abdominal surgery may develop postoperative wound infection and factors commonly associated with wound infection are older age, being underweight, diabetes, contaminated wound, longer preoperative stay. With the aim of reducing the rate of infectious complications, the risk factors can be divided into the following two categories. Non-modifiable factors which include age, contaminated surgical site and the factors that are modifiable before or during surgery are underweight, duration of preoperative and postoperative hospital stay and diabetes mellitus. Further study is required to validate the findings of the present study. Finally, SSI surveillance should be conducted and maintained in all hospitals to promote better surgical outcomes.

\section{REFERENCES}

1. Staneau P, Rioux C, Brucker G. Morbidity and mortality associated with surgical site infections: results from the 1997-1999 INCISO surveillance. J HospInfectc 2001;48: 267-74.

2. Green J, Wand RP, Wenzel. Postoperative wound infection: a controlled study of the increased duration of hospital stay and direct cost of the hospitalization. Ann Surg 1977;185(3):264-68.

3. Mungram AJ, Horan CT, Pearson ML. Guideline for prevention of surgical site infection. Inf Cont Hosp Epid 1999:20(4):247-78.

4. Cruse PJE, Foord R. A five-year prospective study of 23649 surgical wounds. Arch Surg 1973;07:206-9.

5. Howard JM, barker WF, Culbertson WR. Post-operative wound infections: The influence of ultra-violet radiation of the operating rooms and various other factors. Ann Surg $1964 ; 160: 32-81$.

6. Mundy LM, Doherty GM. Inflammation, infection and microbial therapy. In: Doherty GM, editor. Current Surgical Diagnosis and treatment. $13^{\text {th }}$ ed. USA, McGraw-Hill Companies, Inc; 2007. p. 107.

7. Pessaux P, Msika S, Atalla D, Hay JM, Flamant Y. Risk Factors for Postoperative Infectious Complications in Noncolorectal Abdominal Surgery: A Multivariate Analysis Based on a Prospective Multicenter Study of 4718 Patients. Arch Surg 2003;138:314-24.

8. Akihiro W, Kohnoc S, Shimabukuro R, Yamanaka T, Iso Y, Baba H, Higashi H. Risk Factors Associated with Surgical Site Infection in Upper and Lower Gastrointestinal Surgery. Surg 
Today $2008 ; 38: 404-12$.

9. Petrosillo N, Drapeau CMJ, Nicastri E, Martini L, Ippolito G, Moro M, ANIPIO. Surgical Site Infection in Italian Hospital: a Prospective Multicentre Study. BMC Infect Disease 2008;8:34.

10. Cruse PJ, Foord R. A five years prospective study of 23,649 surgical wounds. Arch Surg 1973;107:206-10.

11. Heal C, Buettner P, Browning S. Risk factors for wound infection after minor surgery in general practice. Med J Aust 2006:185(5):255-58

12. Velasco E, Thuler LC, Martins CA, Dias LM, Conalves VM. Risk factors for infectious complications after abdominal surgery for malignant disease. Am J Infect Control 1996;24(1):1-6.

13. Mishriki SF, Law DJW, Jeffery. Factors affecting incidence of Post-operative wound infections. Jour Hosp Infect
$1990: 16(3): 223-30$.

14. Clark MA, Plank LD, Hill GL. Wound Healing Associated with Severe Surgical Illness. World J Surg 2000;24(6):648-54.

15. Moro ML, Carrieri MP, Tozzi AE, Lanas, Greco D. Risk factors for surgical wound infections in clean surgery: a multicenter study. Italian PRINOS Study Group. Ann Ital Chir 1996;67(1):13-9.

16. SSHAIP (ScottishSurveillance of Healthcare Associated Infection Programme). Health Protection Scotland, National Services Division [Internet]. 2005. [Cited 13/07/2016 1:04:05PM] Availablefrom:http://www.hps.scot.nhs.uk/haiic/sshaip/resourcedetail.aspx?id $=743$

17. Claesson BEB, Holmlund DE. Predictors of intraoperative bacterial contamination and postoperative infecton in elective colorectal surgery. J Hosp Infect 1988;11(2):127-35. 